\title{
Growth mechanism of AIN by metal-organic molecular beam epitaxy
}

\author{
I. Gherasoiu, ${ }^{\text {a) }}$ S. Nikishin, G. Kipshidze, B. Borisov, A. Chandolu, C. Ramkumar, \\ M. Holtz, and H. Temkin \\ Department of Electrical Engineering, Texas Tech University, Lubbock, Texas 79409
}

(Received 7 May 2004; accepted 14 September 2004)

\begin{abstract}
The phenomena that accompany the growth of aluminum nitride (AlN) by metal-organic molecular beam epitaxy with trimethylaluminum and ammonia as sources of aluminum and nitrogen, respectively, have been systematically investigated. Optimizing the growth temperature, flux ratios, and the ammonia injector temperature, we obtained an efficient growth with a rate of $500 \mathrm{~nm} / \mathrm{h}$ and a low consumption of ammonia. Layers of AlN with X-ray diffraction linewidth as low as 141 arcsec for the (0002) reflection, and 800 arcsec for the (11-24) reflection are demonstrated on $\mathrm{Si}(111)$ substrates. High temperatures of ammonia injector result in lower growth rates but facilitate transition to the two-dimensional growth. These phenomena are discussed in terms of surface hydrogen, manifested through surfactant effect, and passivation of nitrogen bonds. (C) 2004 American Institute of Physics. [DOI: 10.1063/1.1813623]
\end{abstract}

\section{INTRODUCTION}

Metal-organic chemical-vapor deposition is the technique of choice in the preparation of devices based on IIInitride semiconductors. ${ }^{1}$ Molecular beam epitaxy (MBE), with its relatively simple surface chemistry, offers advantages in terms of growth precision that are important in materials research., ${ }^{2,3}$ Hybrid techniques that combine some of the features of these two methods, such as gas source MBE (GSMBE) with ammonia, have also been used. ${ }^{4,5}$ Growth of gallium nitride $(\mathrm{GaN})$ by a hybrid MBE method in which $\mathrm{Ga}$ is derived from triethylgallium, known as metal-organic molecular beam epitaxy (MOMBE), has been described recently. ${ }^{6}$ The MOMBE method promises a combination of high materials quality, in situ diagnostics, and growth precision that are needed in advanced devices. Despite these potential advantages, very little is known about the MOMBE growth of nitrides. ${ }^{7,8}$

This work presents MOMBE growth of high-quality AIN on $\mathrm{Si}(111)$. Our growth results show the importance of hydrogen in the MOMBE process. AlN is the buffer layer of choice for the growth of GaN, and the AlN crystal quality and surface morphology influence the properties of the entire epitaxial structure. The ability to grow high-quality AlN is thus crucial to these growth methods.

\section{EXPERIMENTS AND RESULTS}

High-quality AlN has been grown on $\mathrm{Si}(111)$ in a compact MOMBE system, ${ }^{9}$ adapted for the growth requirements of nitride-based semiconductors. The sample heater was made out of Ta or $\mathrm{W}$ foil, mounted on a boron nitride plate, allowing growth in the temperature range of $700-900{ }^{\circ} \mathrm{C}$. A $\mathrm{W} / 6 \% \mathrm{Re}-\mathrm{W} / 26 \% \operatorname{Re}$ thermocouple was used to measure the temperature close to the backside of the wafer, while the surface temperature was determined using an infrared pyrometer with spectral sensitivity in the range from

a)Electronic mail: iulian.gherasoiu@ttu.edu
0.89 to $0.95 \mu \mathrm{m}$ and an emissivity setting of $0.68 .{ }^{10}$ The base pressure was $\sim 3 \times 10^{-8}$ Torr, increasing during the growth to as much as $2 \times 10^{-5}$ Torr. A computer-controlled system allows precise temperature and flux profiling during the entire growth cycle.

AlN was grown using trimethylaluminum (TMAl) and $\mathrm{NH}_{3}$ on 50 -mm-diameter $\mathrm{Si}(111)$ substrates. Growth temperatures were in the $760-870{ }^{\circ} \mathrm{C}$ range and beam equivalent pressures (BEP) of TMAl were varied between 3.8 $\times 10^{-6}$ and $7.5 \times 10^{-6}$ Torr. Ammonia was introduced in the growth chamber through an injector containing Mo diaphragms heated to temperatures between 150 and $600{ }^{\circ} \mathrm{C}$. The Mo provides catalytic enhancement for the cracking of ammonia, which would otherwise be negligible in the temperature range employed. ${ }^{11}$

Epitaxial layers were characterized by $x$-ray diffraction (XRD). Surface morphology and microstructure were analyzed using atomic force microscopy measurements and reflection high-energy electron diffraction (RHEED) observations. Strain in AlN was determined through XRD and micro-Raman measurements. Optical reflectivity was used to determine the band gap.

Silicon wafers were prepared using a modified Shiraki etch terminating in a HF:methanol (1:4) rinse. This treatment results in a hydrogen-terminated surface that protects the surface of silicon against reoxidation during handling in air. ${ }^{12}$ Upon the introduction into the growth chamber, the wafer was heated at a rate of $\sim 200{ }^{\circ} \mathrm{C} / \mathrm{min}$ to minimize the exposure time to residual ammonia radicals. Once the wafer reached a temperature of $715 \pm 10{ }^{\circ} \mathrm{C}$, it was exposed for 6-8 s to the TMAl flux admitted into the growth chamber at a BEP of $1.7 \times 10^{-6}$ Torr. The resulting $\mathrm{Al}\left(\mathrm{CH}_{3}\right)_{3}$ coverage further inhibited $\mathrm{Si}$ reactions with residual ammonia. This is supported by experiments on the decomposition of TMAl on $\mathrm{Si}^{13,14}$ A similar procedure was employed by Stevens et al. ${ }^{15}$ in the growth of AlN on $\mathrm{Si}(111)$. The TMAl was turned on again, at a BEP of $3.8 \times 10^{-6}$ Torr, only after the wafer temperature reached $790 \pm 10^{\circ} \mathrm{C}$. Ammonia was admitted into 


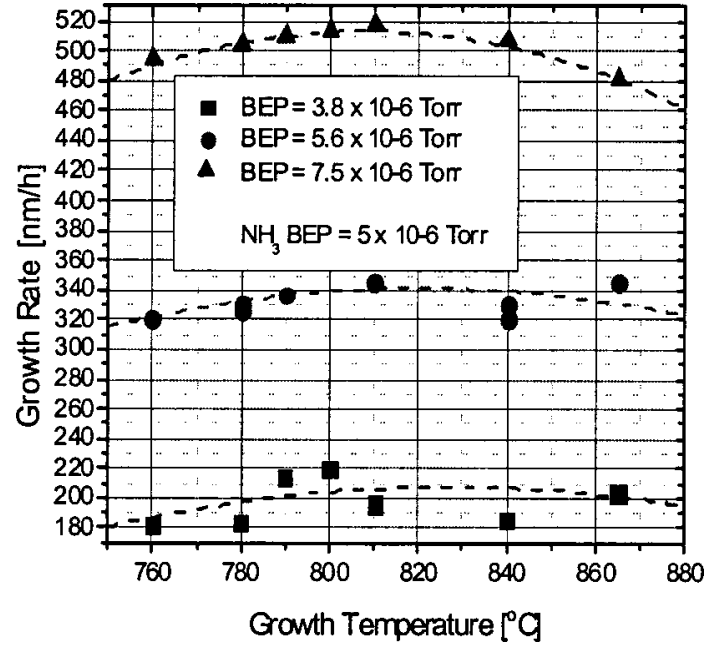

FIG. 1. AlN growth rate as a function of TMAl flux and substrate growth temperature. The lines are guides for eye.

the growth chamber at this point, with a delay of $2-3 \mathrm{~s}$, at a BEP of $4.0 \times 10^{-6}$ Torr. The wafer temperature was ramped in about 6 min to the growth temperature of $860-870{ }^{\circ} \mathrm{C}$. This set of parameters for the initiation of the AlN growth has been selected based on our previous studies of gas source MBE growth, ${ }^{4}$ and optimized for MOMBE. Once the growth temperature stabilized, TMAl flux was readmitted in the chamber and the AIN growth resumed.

Growth rates of AlN are plotted in Fig. 1 as a function of the growth temperature and the TMAl flux, for a constant ammonia BEP of $5.0 \times 10^{-6}$ Torr. This beam pressure of ammonia was obtained at a flow of $35 \mathrm{sccm}$ into the chamber. The injector temperature was kept at $150{ }^{\circ} \mathrm{C}$. In the temperature range of $730-865^{\circ} \mathrm{C}$, the growth rate was weakly temperature dependent with a slight maximum near $800-810^{\circ} \mathrm{C}$. The growth rate scaled with the TMAl flux, increasing from 200 to $520 \mathrm{~nm} / \mathrm{h}$, as the BEP was increased from $3.8 \times 10^{-6}$ to $7.5 \times 10^{-6}$ Torr. The linear dependence on TMAl flux shows that the growth proceeds in the Al-limited regime, and that the nitrogen is released primarily through catalytic dissociation of ammonia in the presence of aluminum. The relative lack of sensitivity of the growth rate on temperature is consistent with previous studies of the growth of AIN by plasma-assisted MBE by Calleja et al. ${ }^{16}$ However, at lower temperatures, around $730{ }^{\circ} \mathrm{C}$, the growth rate was reduced by about a factor of 2 presumably due to lower efficiency of the TMAl decomposition.

The surface roughness of samples grown with low injector temperature exhibited behavior very different from that seen in gas source MBE, i.e., MBE carried out with solid group-III sources and ammonia. There, the surface roughness decreased at higher growth temperatures, consistent with increased surface mobility. In low-temperature MOMBE growth, at $\sim 760{ }^{\circ} \mathrm{C}$, roughness increased from $\sim 12$ to $\sim 23 \AA \mathrm{rms}$ as the flux of TMAl was increased, from 3.8 $\times 10^{-6}$ to $7.5 \times 10^{-6}$ Torr. At high temperatures, $865^{\circ} \mathrm{C}$, the overall roughness increased from $\sim 33$ to $\sim 53 \AA \mathrm{rms}$, as expected.

Figure 2 shows the growth rate of AlN as a function of the flux of ammonia. The experiments included in the data of

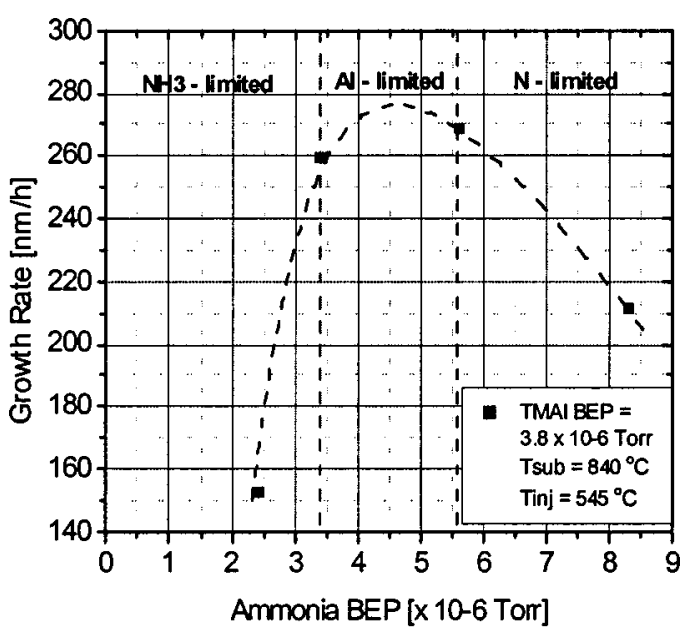

FIG. 2. Growth rate dependence on the $\mathrm{NH}_{3}$ flux for high injector temperature: Tinj $=545^{\circ} \mathrm{C}$. The lines are guides for eye.

Fig. 2 were carried out at the BEP of TMAl of 3.8 $\times 10^{-6}$ Torr and the injector temperature of $545^{\circ} \mathrm{C}$. The growth rate increased initially with the flux of ammonia, saturated, and decreased rapidly with further increases in the flux. This general behavior was observed for all TMAl fluxes used. With lower injector temperatures, the growth rate did not increase over $\sim 200 \mathrm{~nm} / \mathrm{h}$ in the mid-ammonia range, while the decrease at high ammonia fluxes was less pronounced. The behavior observed in Fig. 2 contrasts with the growth rate saturation and plateau seen with the growth with solid group-III source. ${ }^{17,18}$

The MOMBE growth rate of AIN also depends on the temperature of the ammonia injector, as determined for a growth temperature of $865^{\circ} \mathrm{C}$, TMAl BEP of 3.8 $\times 10^{-6}$ Torr, and $\mathrm{NH}_{3} \mathrm{BEP}$ of $8 \times 10^{-6}$ Torr $(47 \mathrm{sccm})$. For injector temperatures up to $200{ }^{\circ} \mathrm{C}$, the growth rate did not change and was $\sim 200 \mathrm{~nm} / \mathrm{h}$. The growth rate of $230 \mathrm{~nm} / \mathrm{h}$ was obtained for injector temperatures of $510-540{ }^{\circ} \mathrm{C}$. For higher injector temperatures, the growth rate decreased markedly, down to $120 \mathrm{~nm} / \mathrm{h}$ at $810^{\circ} \mathrm{C}$.

The injector temperature strongly impacts the growth mode, as seen in RHEED experiments of Fig. 3. All three panels show RHEED images obtained at the end of the growth initiation period, i.e., after $35-60 \mathrm{~nm}$ of growth. Layers of AlN illustrated here were grown at $865{ }^{\circ} \mathrm{C}$, under TMAl BEP of $3.8 \times 10^{-6}$ Torr, and the ammonia pressure of $5 \times 10^{-6}$ Torr. Growth carried out at a low injector temperature $\left(150{ }^{\circ} \mathrm{C}\right)$ always resulted in a spotty, three-dimensional (3D)-like, RHEED pattern, Fig. 3(a). This pattern was seen from the early stages of AlN nucleation, from the first few monolayers. Higher injector temperatures resulted in a gradual transition to the two-dimensional (2D) growth mode. A mixed 3D-2D growth mode could be seen with the injector temperature increased to $410{ }^{\circ} \mathrm{C}$, as shown in Fig. 3(b). Further increase in temperature, to $510^{\circ} \mathrm{C}$, resulted in welldefined 2D growth mode that could be seen from the onset of growth. Figure 3(c) shows a RHEED image obtained on a 35-nm-thick layer of AlN grown with the injector temperature of $510^{\circ} \mathrm{C}$. Well-defined 2D growth was seen on the 

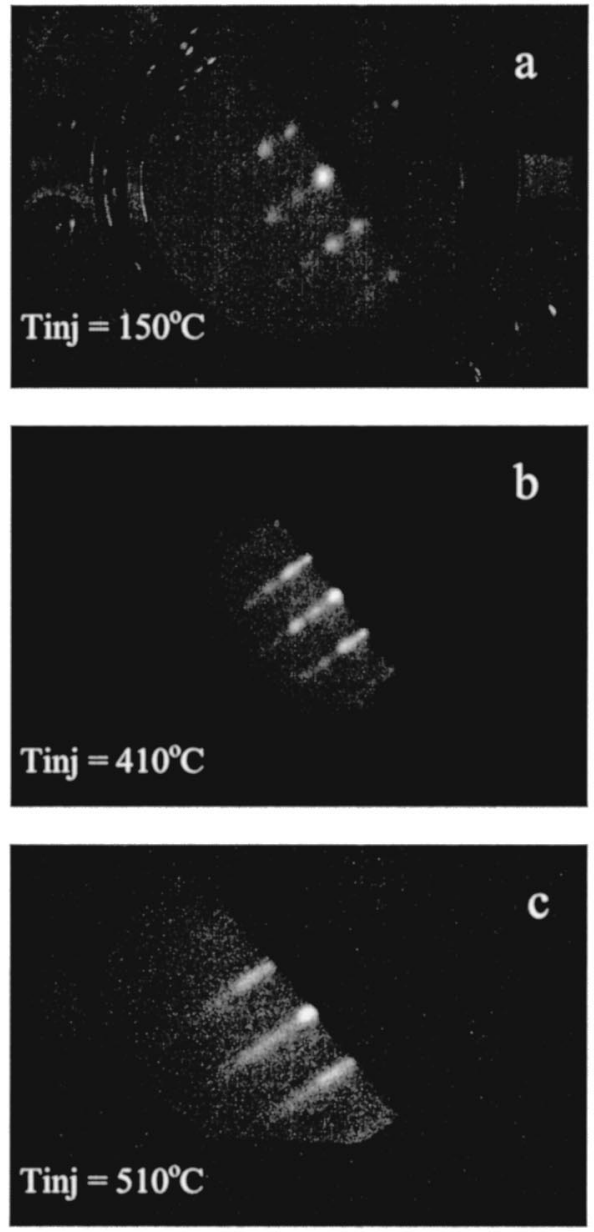

FIG. 3. The change of the RHEED pattern with the increase of the injector temperature.

entire surface, indicating complete coverage of the silicon substrate. The 2D mode was maintained throughout the growth of the 600-nm-thick layer of AlN.

Experiments with a residual gas analyzer, with which our MBE system is equipped, showed that increased injector temperature resulted in release of molecular hydrogen. No cracking of ammonia was observed for injector temperatures up to $300{ }^{\circ} \mathrm{C}$. For the injector temperatures increasing from 300 to $600{ }^{\circ} \mathrm{C}$, the measured partial pressure of molecular hydrogen increased about 15 times, indicating the onset of ammonia cracking. Similar findings regarding catalytic cracking of ammonia in the growth of $\mathrm{GaN}$ were presented by Kamp et al. ${ }^{11}$ Hydrogen thus appears to have a significant role in the $3 \mathrm{D}$ to $2 \mathrm{D}$ growth mode transition observed in MOMBE of AlN.

Figure 4 summarizes the dependence of XRD data on the TMAl flux and growth temperature. The layers with the highest crystalline quality, as measured by the linewidth of the $\omega / 2 \theta$ scan of the (0002) diffraction peak, were prepared at a growth temperature of $780{ }^{\circ} \mathrm{C}$ and a TMAl beam pressure of $5.6 \times 10^{-6}$ Torr. Under these conditions, the (0002) linewidth was only $0.0393^{\circ}$ (or $141.4 \mathrm{arc} \mathrm{sec}$ ), as shown in Fig. 5. To better account for the crystalline structure of this sample, the linewidth of the $\omega$ scan for the (11-24) asymmetric reflection has been measured. Its full width at half maxi-

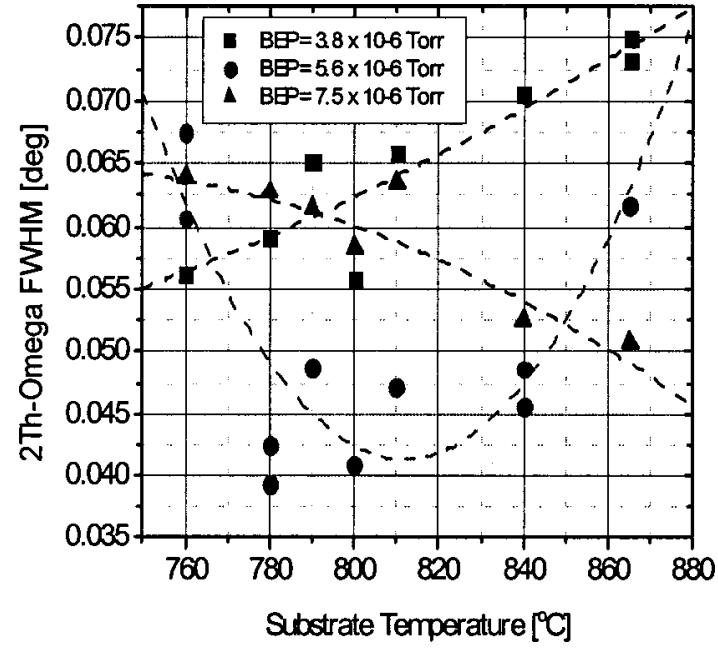

FIG. 4. 2Th-Omega FWHM dependence on TMAl flux and substrate temperature. The lines are guides for eye.

mum (FWHM) was determined to be 800 arc sec. This corresponds to the edge dislocation density of $2.1 \times 10^{9} \mathrm{~cm}^{-2}$.

Figure 4 also plots the XRD linewidths for TMAl beam pressures higher $\left(7.5 \times 10^{-6}\right.$ Torr $)$ and lower $(3.8$ $\times 10^{-6}$ Torr) than the optimum. The linewidth increases (decreases) with temperature for the lower (higher) flux. Since the minimum in the XRD curve is believed to reflect stoichiometric conditions at the growth surface, the data could be interpreted as indicative of a shift in the stoichiometric condition to higher temperatures with increasing flux of TMAl.

The lattice constants of samples grown under stoichiometric conditions were measured from symmetric (0002) and asymmetric (11-24) XRD reflections. For these samples we found the values of lattice parameters $c=4.971 \AA$ and $a$ $=3.121 \AA$ A . Using published lattice constants of bulk AlN,,${ }^{19,20}$ we find the layers under tensile stress in the growth plane, typical for AlN grown on $\mathrm{Si}(111)$. Accordingly, we determine strain in the growth plane to be $\varepsilon_{a} \approx 2.8 \times 10^{-3}$, and the strain along the $c$ axis $\varepsilon_{c} \approx-2.2 \times 10^{-3}$. The corresponding Poisson ratio is then $\nu \approx 0.28$.

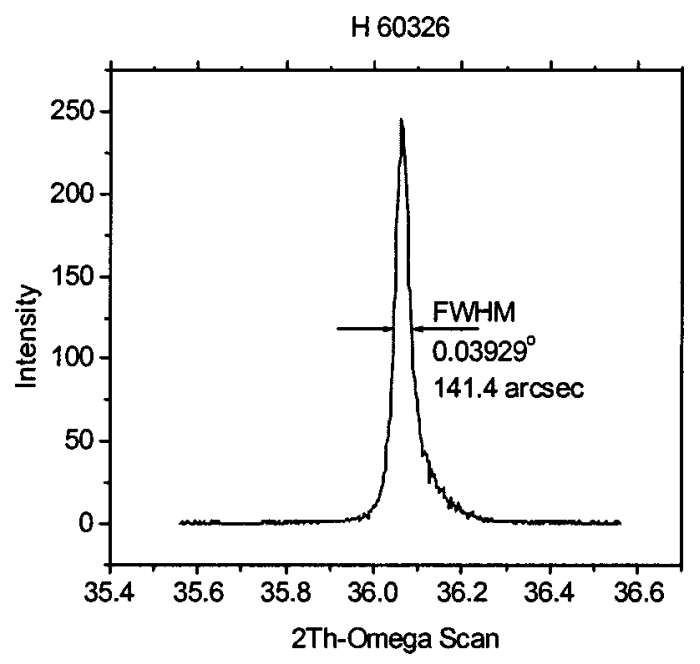

FIG. 5. XRD scans for the best sample grown during the experiment. 
The stress was evaluated by Raman measurements and the values ranged from 0.9 to $1.1 \mathrm{GPa}$, showing no significant lattice relaxation of samples less than $1-\mu \mathrm{m}$ thick. These stresses are in reasonable agreement with the x-ray strain determination, and are attributed to differences in the thermal-expansion coefficients of AlN and silicon, ${ }^{21}$ and residual stress during growth.

Once the optimum parameters, fluxes, and injector temperature were established, high-quality samples with the XRD linewidth below 200 arcsec could be grown reproducibly in a temperature window of about $70-80^{\circ} \mathrm{C}$, i.e., from 780 to $865^{\circ} \mathrm{C}$. The $\omega$ scan of the (0002) diffraction peak, of the sample presented in Fig. 5, showed a FWHM of $0.621^{\circ}$ (37.27'). The lowest FWHM linewidth for the $\omega$ scan, 25.6', was obtained in a sample grown at $800{ }^{\circ} \mathrm{C}$. Lower values $(\omega$ and $\omega / 2 \theta$ scan) have been obtained for the growth of AlN on sapphire and $\mathrm{SiC}$ by plasma-assisted MBE. ${ }^{22-24}$ The best value obtained previously by our group, with gas source MBE on $\mathrm{Si}(111)$, was $\sim 100$ arcsec. $^{21}$

\section{DISCUSSION}

Our MOMBE growth experiments reveal a number of differences with the growth of AlN by gas source MBE. First, we observe a strong decrease in the growth rate at high fluxes of ammonia. This decrease is accelerated with higher injector temperatures. Second, the roughness of AlN samples increases with increasing growth temperature. Third, we observe a $3 \mathrm{D}$ to $2 \mathrm{D}$ transition with increased ammonia injector temperature. We discuss these observations in the context of growth on a hydrogen-passivated surface.

The primary difference between MOMBE and GSMBE has to do with the source of $\mathrm{Al}$. In MOMBE, in order for $\mathrm{Al}$ to bond, it has to lose at least one of the methyl groups. The desorption energy of methyl radicals $E_{\mathrm{des}}\left(\mathrm{CH}_{\mathrm{Al}}\right)$, as determined by Squire et al. ${ }^{25}$ is about $13 \pm 2 \mathrm{kcal} / \mathrm{mol}$. However, such low energy has been observed only at metal surfaces that would easily lose an electron (such as $\mathrm{Al}$ ), or in the presence of more electronegative elements (such as N). On passivated surfaces, the $\mathrm{Al}-\mathrm{C}$ bond breaking energy is much higher, in the range of $50-70 \mathrm{kcal} / \mathrm{mol},{ }^{25,26}$ and $\mathrm{Al}$ can be released only through pyrolitic dissociation. For the range of growth temperatures used in our experiments, the $\mathrm{N}$-stabilized surfaces of $\mathrm{AlN}$, or GaN, are known to be hydrogen passivated and $E_{\mathrm{des}}\left(\mathrm{H}_{\mathrm{N}}\right)=55 \mathrm{kcal} / \mathrm{mol} .^{27}$ The presence of $\mathrm{NH}$ group at the vacuum interface of AlN has been demonstrated by Liu et al. ${ }^{28}$ Their X-ray photoemission spectroscopy study showed that the vacuum interface of AlN, deposited from TMAl and $\mathrm{NH}_{3}$ was $\mathrm{NH}$-terminated up to $\sim 780{ }^{\circ} \mathrm{C}$. Thus, the decomposition of TMAl is unlikely to take place on NH-terminated surface. Instead, Al-stabilized surfaces provide for optimal decomposition of TMAl and $\mathrm{NH}_{3}$ alike, since such surfaces are not passivated by hydrogen within the growth temperature range investigated. The hydrogen desorption energy on such a surface is $E_{\mathrm{des}}\left(\mathrm{H}_{\mathrm{Al}}\right)$ $=17 \mathrm{kcal} / \mathrm{mol},{ }^{27}$ three times lower than that on N-stabilized surface. Assuming stoichiometric growth of AlN and a nitrogen incorporation efficiency of about $10 \%$, we estimate that for $\mathrm{NH}_{3} \mathrm{BEP}$ of $8 \times 10^{-6}$ Torr, the resulting flux impinging the surface would be $\sim 0.4 \times 10^{16} \mathrm{~cm}^{-2} / \mathrm{s}^{-1}$. At growth temperatures around $840{ }^{\circ} \mathrm{C}$, the fractional surface coverage of ammonia would be negligible ${ }^{27}$ and could not constitute a rate-limiting process. On the other hand, hydrogen could provide significant coverage of the growth surface.

The degree of hydrogen passivation, or the fractional surface coverage of hydrogen, is lower at higher temperatures. At high ammonia fluxes, and in particular at high injector temperatures, the hydrogen surface coverage increases resulting in a reduced growth rate. This means that $\mathrm{Al}$ has to compete with the residual hydrogen for the $\mathrm{N}$ bonds at the surface. It is that competition that reduces the growth rate with increased ammonia flux. The three growth regimes apparent in Fig. 2 correspond to different rate-limiting processes at the growth surface. The first region, at low ammonia fluxes, reflects the ammonia-limited growth. In the second region, defined by midrange ammonia fluxes, growth rate is limited by the flux of $\mathrm{Al}$. The growth rate in the third region, of high ammonia fluxes, is limited by the degree of hydrogen passivation and the nitrogen bonds available at the growth surface. This growth region could be called N-limited.

Generally, low roughness of the growth surface is related to high mobility of the species involved and that requires high temperatures. The presence of surfactants, however, can alter such considerations, facilitating a 2D growth at low temperatures. For instance, atomic hydrogen acting as surfactant in the growth of GaAs promotes a 2D growth mode. ${ }^{29}$ The same surfactant effect is observed with the hydrogenassisted growth of $\mathrm{Ge}$ on $\mathrm{Si}(111) .{ }^{30}$ In this case the surface roughness increases at high temperatures, where the fractional surface coverage of hydrogen is lower. This appears to be the case of AlN growth by the MOMBE. There are two major sources of hydrogen in MOMBE growth of AIN. One source is the decomposition of TMAl and $\mathrm{NH}_{3}$ molecules at the surface. Since the growth rate of AlN shown in Fig. 1 is only weakly dependent on temperature, we assume that the cracking rate of source molecules has the same weak dependence. However, at high growth temperatures, the desorption rate of $\mathrm{H}$ increases, resulting in lower fractional surface coverage and increased roughness. The other source of $\mathrm{H}$ is the injector of ammonia. At higher injector temperatures the amount of hydrogen available increases, resulting in a rapid transition to the $2 \mathrm{D}$ growth mode seen in Fig. 3. This observation is also consistent with the H-mediated growth model proposed for GaAs. ${ }^{29}$

\section{CONCLUSION}

In summary, AlN with excellent crystalline quality $(\omega / 2 \theta, 135<$ FWHM $<200$ arcsec $)$ could be obtained by MOMBE under stoichiometric conditions. The mechanisms invoked to account for the MOMBE growth behavior-slow removal of methyl groups, catalytic dissociation of ammonia, and hydrogen passivation of surface nitrogen - can account for increased mobility of $\mathrm{Al}$ on the growth surface, growth rate evolution, and good quality of layers grown at low substrate temperatures. 


\section{ACKNOWLEDGMENTS}

This work was supported by DARPA-SUVOS (Dr. J. Carrano), NSF (ECS-00700240, 0321186, 0304224, and 0323640), NATO SfP 974505, SBCCOM, and the J. F Maddox Foundation.

${ }^{1}$ R. F. Davis, A. M. Roskowski, E. A. Preble, J. S. Speck, B. Heying, J. A. Freitas, Jr., E. R. Glaser, and W. E. Carlos, Proc. IEEE 90, 993 (2002).

${ }^{2}$ H. Markoc, Nitride Semiconductors and Devices (Springer, Heidelberg, 1999).

${ }^{3}$ M. B. Panish and H. Temkin, Gas Source Molecular Beam EpitaxyGrowth and Properties of Phosphorus Containing III-V Heterostructures (Springer, Berlin, 1993).

${ }^{4}$ S. A. Nikishin, et al. Appl. Phys. Lett. 75, 484 (1999).

${ }^{5}$ F. Semond, B. Damilano, S. Ve' zian, N. Grandjean, M. Leroux, and J. Massies, Appl. Phys. Lett. 75, 82 (1999).

${ }^{6}$ T. Li, R. P. Campion, C. T. Foxon, S. A. Rushworth, and L. M. Smith, J. Cryst. Growth 251, 499 (2003).

${ }^{7}$ J. D. MacKenzie, C. R. Abernathy, S. J. Pearton, V. Krishnamoorthy, S. Bharatan, K. S. Jones, and R. G. Wilson, Appl. Phys. Lett. 67, 253 (1995).

${ }^{8}$ S. C. Jain, M. Willander, J. Narayn, and R. Van Overstraeten, Appl. Phys. Lett. 87, 965 (2000)

${ }^{9}$ R. A. Hamm, D. Ritter, and H. Temkin, J. Vac. Sci. Technol. A 12, 2790 (1994).

${ }^{10}$ S.S. Nikishin, S. Francoeur, and H. Temkin, Mater. Res. Soc. Symp. Proc. 639, G.6.57. p.5 (2001)

${ }^{11}$ M. Kamp, M. Mayer, A. Pelzmann, and K. J. Ebeling, MRS Internet J. Nitride Semicond. Res. 2, 26 (1997).

${ }^{12}$ G. S. Higashi, Y. J. Chabal, G. W. Trucks, and K. Raghavachari, Appl. Phys. Lett. 56, 656 (1990).
${ }^{13}$ M. M. Frank et al., Appl. Phys. Lett. 83, 740 (2003).

${ }^{14}$ M. Ishida, A. Eto, and T. Nakamura, J. Vac. Sci. Technol. A 7, 2931 (1989).

${ }^{15}$ K. S. Stevens, A. Ohtani, M. Kinniburgh, and R. Beresford, Appl. Phys. Lett. 65, 321 (1994).

${ }^{16}$ E. Calleja, M. A. Sanchez-Garcia, E. Monroy, F. J. Sanchez, and E. Munoz, J. Appl. Phys. 82, 4681 (1997).

${ }^{17}$ S. Nikishin et al., J. Vac. Sci. Technol. B 19, 1409 (2001).

${ }^{18}$ M. Mesrine, N. Grandjean, and J. Massies, Appl. Phys. Lett. 72, 350 (1998).

${ }^{19}$ I. Vurgaftman and J. R. Meyer, J. Appl. Phys. 94, 3675 (2003).

${ }^{20}$ Properties of advanced semiconductor materials GaN, AlN, InN, BN, SiC, and SiGe, edited by M. E. Levinshtein, S. L. Rumyantsev, and M. S. Shur (Wiley, New York, 2001).

${ }^{21}$ T. Prokofyeva, M. Seon, J. Vanbuskirk, M. Holtz, S. A. Nikishin, N. N. Faleev, H. Temkin, and S. Zollner, Phys. Rev. B 63, 125313 (2001).

${ }^{22}$ K. Jeganathan, T. Kitamura, M. Shimizu, and H. Okumura, Jpn. J. Appl. Phys., Part 2 41, L28 (2002).

${ }^{23}$ N. Onojima, J. Suda, and H. Matsunami, Jpn. J. Appl. Phys., Part 2 42, L445 (2003).

${ }^{24}$ X. Q. Shen, M. Shimizu, and H. Okumura, Jpn. J. Appl. Phys., Part 242 , L1293 (2003).

${ }^{25}$ D. W. Squire, C. S. Dulcey, and M. C. Lin, J. Vac. Sci. Technol. B 3,1513 (1985).

${ }^{26}$ G. V. Jagannathan, M. L. Andrews, and A. T. Habig, Appl. Phys. Lett. 56, 2019 (1990).

${ }^{27}$ S. W. King, E. P. Carlson, R. J. Therrien, J. A. Christman, R. J. Nemanich, and R. F. Davis, J. Appl. Phys. 10, 5584 (1999).

${ }^{28}$ H. Liu, D. C. Bertolet, and J. W. Rogers, Jr., Surf. Sci. 340, 88 (1995).

${ }^{29}$ Y. Okada and J. S. Harris, Jr., J. Vac. Sci. Technol. B 14, 1725 (1996).

${ }^{30}$ A. Sakai and T. Tatsumi, Appl. Phys. Lett. 64, 52 (1994). 